Pacific Journal of Mathematic 


\title{
$d$-SIMPLE SETS, SMALL SETS, AND DEGREE CLASSES
}

\author{
MANuel Lerman AND Robert I. SOARE
}

\begin{abstract}
A new notion of simplicity for recursively enumerable (r.e.) sets is introduced, that of $d$-simplicity or simplicity with respect to arrays of differences of r.e. sets (d.r.e. sets). This notion arose from the method used to generate automorphisms of $\mathscr{E} *$, the lattice of r.e. sets modulo finite sets, and is a further step toward finding a complete set of invariants for the automorphism types of $\mathscr{E} *$. The $d$ simple sets are closely related to the small sets defined by Lachlan as a key part of his decision procedure for the $\forall \exists$-theory of $\mathscr{E}^{*}$. Finally, the degrees $D$ of $d$-simple sets form a new invariant class of r.e. degrees, since $H_{1} \subseteq D$ but $D$ splits $L_{1}$ (where $H_{1}$ and $L_{1}$ are the high and low r.e. degrees respectively). This refutes conjectures of Martin and Shoenfield which imply that degrees $C$ of any class of r.e. sets invariant under automorphisms of $\mathscr{E}$ can be characterized by a finite set of equalities or inequalities involving the jump of degrees in $C$.
\end{abstract}

o. Introduction. Let $\mathscr{E}$ denote the lattice of r.e. sets under inclusion. If $\mathcal{C}$ is a sublattice of $\mathscr{E}$ closed under finite differences, let $\mathscr{P}^{*}$ denote the quotient lattice of $\mathscr{P}$ modulo the ideal $\mathscr{F}$ of finite sets. Post's program [11] which has predominated for thirty years has been to classify an r.e. set $A$ by its lattice of supersets $\gamma(A)=\{W: W \in \mathscr{E}$ and $A \subseteq W\}$. Further evidence for this approach was the automorphism result by Soare [17] that if $A$ and $B$ are maximal sets (i.e., $\mathscr{C}^{*}(A)$ and $\mathscr{C}^{*}(B)$ are isomorphic to the two element Boolean algebra) then $A$ and $B$ are automorphic, i.e., there exists $\Phi \in$ Aut $\mathscr{E}$ (the group of automorphisms of $\mathscr{E}$ ) such that $\Phi(A)=B$.

However, more recent results [9] show that $\mathscr{P}^{*}(A) \cong \mathscr{L}^{*}(B)$ does not necessarily imply that $A$ is automorphic to $B$ when $\mathscr{Z}^{*}(A)$ is infinite, even if $\mathcal{C}^{*}(A)$ is a very well-behaved lattice such as the countable atomless Boolean algbera. To characterize the automorphism type of $A \in \mathscr{E}$ new invariants are needed which, unlike $P^{*}(A)$, relate the structure of $\bar{A}$ to that of $A$. (Warning: all sets and degrees mentioned will be r.e.)

A second automorphism result [20] demonstrating uniformity of $\mathscr{C}^{*}$ is that if $A$ is coinfinite and low (i.e., $\left.A^{\prime} \equiv{ }_{T} \phi^{\prime}\right)$ then $\mathscr{L}^{*}(A) \cong$ $6^{*}$, and in fact the isomorphism is effective on indices. If $A$ and $B$ are low simple sets are they necessarily automorphic? In order to extend an automorphism $\Psi: \mathscr{P}^{*}(A) \rightarrow \mathscr{P}^{*}(B)$ to an automorphism 
$\Phi$ of $\mathscr{E}^{*}$ such that $\Phi(A)=\Phi(B)$, the automorphism method uses a certain covering property [17, Theorem 2.2] of which the notion of $d$-simplicity, defined below, is a weak version. We prove that there are low simple sets $A$ and $B$ such that $A$ is $d$-simple but $B$ is not, and hence $A$ is not automorphic to $B$. Thus, $d$-simplicity is a new lattice invariant property of sets $A \in \mathscr{E}$ not definable in terms of $\mathscr{P}^{*}(A)$.

A class $\mathscr{C} \subseteq \mathscr{E}$ is invariant if it is invariant under Aut $\mathscr{E}$. A class $C$ of degrees is invariant if $C=\{\operatorname{deg}(W): W \in \mathscr{C}\}$ for some invariant $\mathscr{C} \subseteq \mathscr{E}$. A fundamental open question relating the structure of a set to its degree is to determine which classes of degrees are invariant. Let $\boldsymbol{R}$ denote the (r.e.) degrees and define

$$
\begin{aligned}
& \boldsymbol{H}_{n}=\left\{\boldsymbol{a}: \boldsymbol{a} \in \boldsymbol{R} \text { and } \boldsymbol{a}^{(n)}=\mathbf{0}^{(n+1)}\right\} . \\
& \boldsymbol{L}_{n}=\left\{\boldsymbol{a}: \boldsymbol{a} \in \boldsymbol{R} \text { and } \boldsymbol{a}^{(n)}=\mathbf{0}^{(n)}\right\},
\end{aligned}
$$

where $\boldsymbol{d}^{(0)}=\mathbf{0}$, and $\overline{\boldsymbol{L}}_{n}=\boldsymbol{R}-\boldsymbol{L}_{n}$. The degrees in $\boldsymbol{H}_{1}$ and $\boldsymbol{L}_{1}$ are called high and low respectively. Martin [9] showed that $\boldsymbol{H}_{1}=\boldsymbol{M}$, the degrees of maximal sets and Lachlan [4] and Shoenfield [16] proved that $\overline{\boldsymbol{L}}_{2}=\boldsymbol{A}$, the degrees of atomless sets. Given this progression of invariant classes, $\overline{\boldsymbol{L}}_{0}, \boldsymbol{H}_{1}, \overline{\boldsymbol{L}}_{2}$, Shoenfield conjectured that these exhausted the invariant classes while Martin conjectured that the invariant classes are precisely $\overline{\boldsymbol{L}}_{2 n}$ and $\boldsymbol{H}_{2 n+1}$ for $n \geqq 0$.

The major achievement of this paper is to prove that $\boldsymbol{D}$, the class of degrees of $d$-simple sets, is a new invariant class not of the form $\boldsymbol{H}_{n}$ or $\ddot{\boldsymbol{L}}_{n}$ for any $n$. This is accomplished by showing that $\boldsymbol{H}_{1} \subseteq \boldsymbol{D}$, but that $\boldsymbol{D}$ splits $\boldsymbol{L}_{1}$ and in fact that there is a simple set $S$ with no $d$-simple set recursive in $S$. The other known classes of r.e. sets which contain members of some degree $\boldsymbol{d} \in \overline{\boldsymbol{L}}_{1}-\boldsymbol{L}_{0}$ (such as simple or hypersimple sets) can be shown to contain members of every r.e. degree $\boldsymbol{d}>\mathbf{0}$ using the permitting method of Yates [22]. Such methods fail here because $d$-simplicity is defined in terms of certain arrays of differences of r.e. sets (d.r.e. sets) rather than arrays of r.e. sets.

The plan of the paper is as follows. In $\S 1$ we define $d$-simplicity and prove that hyperhypersimple (hh-simple) sets are $d$-simple and that $d$-simple sets are simple. We prove that the class $\mathscr{D}$ of $d$-simple sets is closed upwards under inclusion (among the coinfinite sets) and that $\boldsymbol{D}$ is closed upwards and $\boldsymbol{H}_{1} \subseteq \boldsymbol{D}$. Finally, we prove that there are low $d$-simple sets so $\boldsymbol{D} \cap \boldsymbol{L}_{1} \neq \phi$.

In $\S 2$ we review the small sets introduced by Lachlan [3] in his decision procedure for the $\forall \exists$-theory of $\mathscr{E}^{*}$. We prove that no $d$-simple set is small, that there is a simple small (and hence not 
$d$-simple) set in every degree $\boldsymbol{d}>\mathbf{0}$, and that the $d$-simple sets do not coincide with any of the well-known classes of simple sets. There is a coinfinite r.e. set with no $d$-simple superset and the class of degrees of such sets is exactly $\boldsymbol{H}_{1}$.

In $\S 3$ we prove that the $d$-simple sets are not closed under intersection and that the relation " $d$-simple in" is not transitive. In $\S 4$ we prove our most important and difficult result that there is a degree $\boldsymbol{d} \in \boldsymbol{L}_{1}$ such that all sets of degree $\leqq \boldsymbol{d}$ are small and hence not $d$-simple. Thus $D$ splits $L_{1}$.

We use the standard notation in Rogers [14]. In addition let $A={ }^{*} B$ denote that the symmetric difference of $A$ and $B$ is finite, and $A \subseteq{ }^{*} B$ denote that $A \cap \bar{B}={ }^{*} \phi$. Let $B \subset{ }_{\infty} A$ denote that $A \subseteq B$ and $A-B$ is infinite. A simultaneous enumeration of a given recursive sequence $\left\{U_{n}\right\}_{n \in N}$ is a $1: 1$ recursive function $g$ with range $\left\{\langle m, n\rangle: m \in U_{n}\right\}$. Thus, at each stage $s, g(s)=\langle m, n\rangle$ causes one element $m$ to be enumerated in one set $U_{n}$. Fixing $g$ let $U_{n}^{s}$ denote those elements enumerated in $U_{n}$ by the end of stage $s$, and

$$
U_{n} \backslash U_{m}=\left\{x:(\exists s)\left[x \in U_{n}^{s}-U_{m}^{s}\right]\right\},
$$

those elements appearing in $U_{n}$ before $U_{m}$. (The notation $X \backslash Y$ should not be confused with $X-Y$ which denotes $X \cap \bar{Y}$.) Let $U_{n} \searrow U_{m}=\left(U_{n} \backslash U_{m}\right) \cap U_{m}$, those elements enumerated first in $U_{n}$ and later in $U_{m}$.

We identify a set with its characteristic function and let $A[x]$ denote the restriction of $A$ to arguments $\leqq x$. We write $\{e\}_{s}^{A[z]}(x)=$ $y$ if the eth Turing procedure with argument $x$ and oracle $A[z]$ halts in $\leqq s$ steps and yields output $y$. We assume that $e, x, y, z \leqq$ $s$, that $x, y$, $\leqq z$, and that if $z_{1} \leqq z$ is used in the computation then all $z_{2} \leqq z_{1}$ are also used.

1. $d$-simple sets. We begin with a motivation of $d$-simplicity from the point of view of generating automorphisms of $\mathscr{E}$. Suppose that $A$ and $B$ are coinfinite low sets (so $\mathscr{L}(A) \cong \mathscr{L}(B)$ by [20]). Let us try to construct $\Phi \in$ Aut $\mathscr{E}$ such that $\Phi(A)=B$ by enumerating an array $\left\{\hat{W}_{e}\right\}_{e \in N}$ such that $\Phi\left(W_{e}\right)=\hat{W}_{e}$. Now if $B$ is simple and $A$ is not, say $W_{e} \cap A=\phi$, for $W_{e}$ infinite, then we must fail since $\hat{W}_{e} \cap B \neq \phi$ for any choice of $\hat{W}_{e}$ infinite. To avoid this problem suppose that $A$ and $B$ are simple.

Given $W_{1} \supseteqq W_{2}$ which intersect $\bar{A}$ we must choose $\hat{W}_{1} \supseteqq \hat{W}_{2}$ which intersect $\bar{B}$. If we choose $\hat{W}_{1}$ and $\hat{W}_{2}$ such that $\left(\hat{W}_{1}-\hat{W}_{2}\right) \cap$ $B \neq \phi$ then we must be sure that $A$ is sufficiently "large" so that $A \cap\left(W_{1}-W_{2}\right) \neq \phi$ also, i.e., if $B$ is simple with respect to certain arrays of d.r.e. sets then $A$ must also be. What is the right defini- 
tion of simplicity with respect to d.r.e. sets? Clearly we cannot ask that $A$ intersect every infinite d.r.e. set since $\bar{A}$ is d.r.e. and infinite.

The definition is motivated by the hypotheses of the Extension Theorem [17, Theorem 2.2] for generating automorphisms of $\mathscr{E}$, where the problem of defining $\hat{X}=\Phi(X)$ is split into 2 parts corresponding to $X \cap \bar{A}$ and $X \cap A$. First consider $X \cap \bar{A}$ and choose a set $Y \subseteq X$ as small as possible such that $Y \cap \bar{A}=X \cap \bar{A}$. Then consider $X \cap A$ and let $\hat{X}$ be sufficiently large so that $\hat{X} \cap B \supseteq \hat{Y} \cap B$.

Definition 1.1. (a) A coinfinite set $A$ is $d$-simple if for all $X$ there exists $Y \subseteq X$ such that

(1.1) $X \cap \bar{A}=Y \cap \bar{A}$, and

(1.2) $(\forall Z)[(Z-X)$ infinite $\Rightarrow(Z-Y) \cap A \neq \phi]$.

(b) Furthermore, $A$ is uniformly $d$-simple if an index for $Y$ can be found uniformly effectively from one for $X$.

Note that (1.2) asserts that $A$ is simple with respect to the r.e. array of d.r.e. sets $\left\{\left(W_{e}-Y\right)\right\}_{e \in \omega}$. (Of course, $d$-simplicity is definable in the elementary theory of $\mathscr{E}$ and hence invariant under Aut $\mathscr{E}$.)

Proposition 1.2. If $A$ is $d$-simple then $A$ is simple.

Proof. In Definition 1.1 set $X=\dot{\phi}$. Hence $Y=\dot{\phi}$ and (1.2) asserts that $Z \cap A \neq \phi$ for every infinite $Z$.

Proposition 1.3. If $A$ is hh-simple then $A$ is $d$-simple.

Proof. Lachlan [2, Theorem 3] has shown that a coinfinite set $A$ is $h h$-simple iff $\mathscr{P}(A)$ is a Boolean algebra or equivalently iff for every $X$ there is a recursive $R \subseteq X$ such that $X \cap \bar{A}=R \cap \bar{A}$. In (1.1) set $Y=R$. Now if $Z$ violates (1.2) then $Z-R$ is infinite but $(Z-R) \cap A=\phi$, so $Z \cap \bar{R}$ is an infinite r.e. subset of $\bar{A}$ violating the simplicity of $A$.

Notice that Proposition 1.3 does not establish that $h h$-simple sets are uniformly $d$-simple since Lachlan's procedure gives us no uniform way of finding $R$ from $X$.

Proposition 1.4. Let $\mathscr{D} \subseteq \mathscr{E}$ be the class of $d$-simple sets. Then $\mathscr{D}$ is closed upwards among the coinfinite sets, namely if $A \in \mathscr{D}$ and $A \subseteq B \subset_{\infty} N$ then $B \in \mathscr{D}$ also.

Proof. If (1.1) and (1.2) hold for $A$ then they hold a fortiori for $B$ since (1.1) for $\bar{A}$ and $\bar{B} \subseteq \bar{A}$ imply (1.1) for $\bar{B}$, while (1.2) for 
$A$ and $A \subseteq B$ imply (1.2) for $B$.

Proposition 1.5. If $\mathscr{6} \subseteq \mathscr{E}$ is a class of coinfinite sets, and is closed upwards among the coinfinite sets, and $\mathscr{C}$ contains all hh-simple sets then $C=\{\operatorname{deg}(W): W \in G\}$ is closed upwards and $\boldsymbol{C} \supseteq \boldsymbol{H}_{1}$.

Proof. Martin [10] shows that every $\boldsymbol{d} \in \boldsymbol{H}_{1}$ contains a maximal (and hence $h h$-simple) set. Hence $\boldsymbol{H}_{1} \leqq C$. Now suppose $\boldsymbol{a}>\boldsymbol{b}$, and $\boldsymbol{b} \notin \boldsymbol{H}_{1}$ where $\boldsymbol{b}=\operatorname{deg} B$ and $B \in{ }^{\prime} \boldsymbol{C}$. Then by Martin [10] $B$ is not hh-simple so by Lachlan [4, Theorem 1] there exists $A \supseteqq B$ of degree $\boldsymbol{a}$. Now $A \in \%$ by upward closure so $\boldsymbol{a} \in \boldsymbol{C}$.

Corollary 1.6. Let $\boldsymbol{D}=\{\operatorname{deg}(W): W \in \mathscr{D}\}$. Then $\boldsymbol{H}_{1} \leqq \boldsymbol{D}$ and $D$ is closed upwards.

Proof. By Propositions 1.5., 1.4 and 1.3.

Next we prove that there is a low $d$-simple set, and hence $\boldsymbol{D} \cap \boldsymbol{L}_{1} \neq \phi$. The construction is very similar to the usual construction [18, Theorem 4.1] of a low simple set $A$ except that $A$ must now intersect certain infinite d.r.e. sets instead of certain infinite r.e. sets. Let $\left\{\left\langle X_{e}, Z_{e}\right\rangle\right\}_{e \in \omega}$ be a recursive listing of all pairs of r.e. sets, and fix a simultaneous enumeration of $\left\{X_{e}, Z_{e}\right\}_{e \in \omega}$. To make $A d$-simple it suffices to make $\bar{A}$ infinite and to meet for each $e$ the positive requirement,

$$
P_{e}:\left(Z_{e}-X_{e}\right) \text { infinite } \Longrightarrow(\exists x)(\exists s)\left\lfloor x \in\left(Z_{e}^{s}-X_{e}^{s}\right) \cap\left(A^{s+1}-A^{s}\right)\right\rfloor,
$$

because we can let $Y_{e}=X_{e} \backslash A$. (Recall that $U \backslash V=\left\{x:(\exists s)\left\lfloor x \in U^{s}-\right.\right.$ $\left.V^{s} \mid\right\}$.) Thus $Y_{e}$ satisfies (1.1) because $X_{e} \cap \bar{A} \subseteq X_{e} \backslash A$, and (1.2) holds because an element $x \in Z_{e}^{s}-X_{e}^{s}$ enumerated in $A$ at stage $s+1$ is never later enumerated in $Y_{e}$ so $\left(Z_{e}-Y_{\rho}\right) \cap A \neq \phi$.

Each $P_{c}$ contributes at most one element to $A$, and the lowness requirements $N_{e}$ defined below involve finite restraint, so the usual construction succeeds. Finally, the uniformity condition (b) of Definition 1.1 is satisfied by the definition of $Y_{e}$.

TheORem 1.7. There is a low uniformly d-simple set $A$.

Proof. To make $A$ low it suffices [18, p. 523] to meet for each $e$ the negative requirement,

$N_{e}:\{e\}_{s}^{A^{s}}(e)$ defined for infinitely many $s \Longrightarrow\{e\}^{-1}(e)$ defined.

Let $r(e, s)$ be the greatest integer used in the computation $\{e\}_{s}^{A_{s}}(e)$ 
if the latter is defined and $=-1$ otherwise. Set $A^{0}=\phi$.

Stage $s+1$. Choose $e$ minimal such that $P_{e}$ has never received attention and such that

$$
(\exists x)\left[x \in Z_{e}^{s}-\left(X_{e}^{s} \cup A^{s}\right) \& 2 e<x \&(\forall i \leqq e)[r(i, s)<x]\right] .
$$

Choose $x$ minimal for $e$. Enumerate $x$ in $A$ and say that $P_{e}$ receives attention. (If $e$ fails to exist do nothing.) Let $A=\mathrm{U}_{s} A^{s}$.

The second clause in (1.4) guarantees that $\bar{A}$ is infinite. Each requirement $N_{e}$ is met because each $P_{i}, i<e$, contributes at most one element to $A$. Thus, $\lim \sup _{s} r(i, s)$ exists for all $i$ and each requirement $P_{e}$ is met.

\section{CoROLlaRy 1.8. $\quad D \cap \boldsymbol{L}_{1} \neq \dot{\phi}$.}

Our results yield new negative information on the question of what conditions on $A$ and $B$ guarantee that

$$
\mathscr{L}(A) \cong \mathscr{L}(B) \Longrightarrow A \text { is automorphic to } B \text {. }
$$

Let $A$ be low and $d$-simple, and $B$ be the low simple set of Corollary 2.7 which by Proposition 2.3 is not $d$-simple. Now $\mathscr{P}(A) \cong \mathscr{L}(B) \cong$ $\mathscr{E}$ by [19] but $A$ and $B$ are not automorphic, because $d$-simplicity is clearly invariant under Aut $\mathscr{E}$. Hence, (1.5) is false for low simple sets and the $d$-simplicity of an r.e. set $A$ is not definable as a property of $\mathscr{L}(A)$. In [9] it is shown that (1.5) is false for atomless $h h$-simple sets. It is unknown whether (1.5) holds when $A$ and $B$ are both low and $d$-simple but this seems unlikely. Maximal sets satisfy (1.5) because they possess a stronger covering property than $d$-simplicity $[17, \S 3]$. It is unknown whether this stronger property is invariant under Aut $\mathscr{E}$ or under what conditions it is implied by $d$-simplicity. However, the construction of Theorem 1.7 can easily be modified to produce low simple sets with the stronger property.

2. Small sets. A second notion which relates the structure of $A$ to that of $\bar{A}$ (and is not merely a property of $\mathscr{L}(A)$ ) is the notion of a small set introduced by Lachlan [3, Theorem 3] as an important ingredient in his decision procedure. In this section we prove that no $d$-simple set is small, that there is a simple small (and hence not $d$-simple) set in every degree $\boldsymbol{d}>\mathbf{0}$, and that the $d$-simple sets do not coincide with any other well-known classes of simple sets.

Definition 2.1. (a) If $B \subset A \subset_{\infty} N$ then $B$ is small in $A\left(B \subset_{8} A\right)$ if for all $U$ and $V$ 


$$
V \supseteqq U \cap(A-B) \Longrightarrow(U-A) \cup V \text { is r.e. }
$$

(b) $B$ is small if $B C_{s} A$ for some $A$.

The intuition is that $B$ is sufficiently smaller than $A$ so that any $V$ satisfying the hypothesis of (2.1) must include enough of $U$ so that the union of the d.r.e. set $(U-A)$ with $V$ is r.e. Notice that $\phi \subset_{s} A$ for every $A \subset_{\infty} N$ (because $(U-A) \cup V=U \cup V$ ), and for $A$ nonrecursive no $B={ }^{*} A$ is small in $A$. (If so set $U=N$, and $V=A-B$ implying that $\bar{A}$ is r.e.) The terminology "small" was introduced by M. Stob [21] after he observed,

Proposition 2.2. (Stob). (a) If $A \subset B \subset C \subset_{\infty} N$ and either $A \subset_{s} B$ or $B \subset_{s} C$ then $A \subset_{s} C$.

(b) If $A \subset_{\infty} C \subset_{\infty} N$ and $A \cup \bar{C}$ is not r.e., and $A$ is not recursive, then there exists $B$ such that $A \subset B \subset C, A \not \subset s B$, and $B \not \subset_{s} C$.

Part (a) asserts not only that $C_{s}$ is transitive but also that small sets are closed downwards under inclusion, while (b) implies that no notion of $A$ being "close" to $C$ can force all intermediate sets $B$ to be small in $C$.

Proposition 2.3. If $B$ is small then $B$ is not $d$-simple.

Proof. Let $B \subset_{s} A \subset_{\infty} N$. Suppose that $B$ is $d$-simple. Then by Definition 1.1 with $X=A$ there must exist $Y \subseteq X$ such that

$$
\begin{gathered}
X \cap \bar{B}=Y \cap \bar{B} \text {, and } \\
(\forall Z)[(Z-X) \text { infinite } \Longrightarrow(Z-Y) \cap B \neq \phi] .
\end{gathered}
$$

Now in Definition 2.1 set $U=N$ and $V=Y$. By (2.2), $V \supseteqq U \cap$ $(A-B)$ and hence $(U-A) \cup Y$ is r.e. by (2.1). But then $Z=$ $(U-A) \cup Y$ violates $(2.3)$ because $(Z-Y)=(N-A)$ is infinite but fails to intersect $B$.

M. Stob [21] and, independently, E. Herrmann have shown the converse to be false by producing a simple set which is neither small nor $d$-simple. Essentially the same proof as in Proposition 2.3 establishes the following alternate characterization of $d$-simple sets which emphasizes their relationship to small sets.

Proposition 2.4. If $A \subset_{\infty} N$ then $A$ is $d$-simple if and only if for all $X \supseteqq A$ there exists $Y$ such that

$$
X \cap \bar{A}=Y \cap \bar{A}, \text { and }
$$



$\neg(\exists W)[(W-X)$ infinite and $(W-X) \cup Y$ is r.e. $]$.

To show that every degree $\boldsymbol{d}>\mathbf{0}$ contains a simple small (and hence not $d$-simple) set we recall some well-known results.

Proposition 2.5. For any simple set $S$ and degree $\boldsymbol{d}>0$ there is a simple set $A \subseteq S$ of degree $\boldsymbol{d}$.

Proof. See either [18, Theorem 3.10] or [8, Theorem 3.1].

Definition 2.6. If $B \subset_{\infty} A$ then $B$ is a major subset of $A$ $\left(B \subset_{m} A\right)$ if for all $W$,

$$
W \cup A={ }^{*} N \Longrightarrow W \cup B={ }^{*} N .
$$

Lachlan [3, Theorem 3] proved that every nonrecursive r.e. set $A$ has a major subset $B$ such that $B \subset_{s} A$ (written $B \subset_{s m} A$ ). Notice that the requirements of majoricity and smallness tend to conflict because $B \subset_{m} A$ requires $B$ "close" to $A$ while $B C_{s} A$ requires $B$ "far away" from $A$.

CoRollary 2.7. For any simple set $A$ and degree $\boldsymbol{d}>\mathbf{0}$ there is a small simple set $B \subseteq A$ of degree $\boldsymbol{d}$.

Proof. Given $A$ simple find $M \subset_{s m} A$ by Lachlan [3, Theorem 3] and simple $B \subset M$ of degree $d$ by Proposition 2.5. Now $B \subset_{s} A$ by Proposition 2.2 (a).

(Of course the result for $B$ merely non $d$-simple and not necessarily small follows by the same proof without Proposition 2.2 and the notion of smallness using the downward closure of non- $d$-simple sets of Proposition 1.4.)

Notice that $\mathscr{D}$ does not coincide with any of the well-known classes of simple sets such as simple, hypersimple, $h h$-simple, or $r$-maximal, etc. (A coinfinite set $A$ is $r$-maximal if there is no recursive set $R$ such that $R \cap \bar{A}$ and $\bar{R} \cap \bar{A}$ are both infinite.) Other classes of simple sets are discussed in $[19, \S 3]$. All of these but the simple and hypersimple sets exist only in high degrees while Theorem 1.7 produced a $d$-simple set which is both low and not hypersimple [14, p. 138] by the second clause of (1.4). Thus $\mathscr{D}$ is contained in the simple sets but in no other of the usual classes.

Furthermore, $\mathscr{D}$ does not contain any of these classes except for the $h h$-simple sets. Proper $r$-maximal ( $r$-maximal, nonmaximal) sets may be either $d$-simple or not. If $A$ is $r$-maximal and $B \subset_{s m} A$ 
then $B$ is also $r$-maximal but small and hence not $d$-simple. On the other hand the usual constructions [5, p. 300] or [12, Theorem 6] of atomless $r$-maximal sets can easily be combined with the positive requirements $P_{e}$ of Theorem 1.7 to produce an atomless $r$-maximal $d$-simple set. (We say that $A$ is atomless if $A$ has no maximal superset.)

R.A. Shore and the authors have noted that the Robinson construction [12, Theorem 6] and the Lachlan small major subset construction [3, Theorem 3] may be combined to produce a "small tower" $\left\{A_{n}\right\}_{n \in \omega}$ of simple sets such that

$$
(\forall i)\left[A_{i} \subseteq_{8} A_{i+1} \subset_{\infty} N\right],
$$

and

$$
(\forall W)\left[\bar{A}_{0} \subset^{*} W \text { or }(\exists i)\left[W \subseteq A_{i}\right]\right] .
$$

Condition (2.7) guarantees that $A_{0}$ is $r$-maximal and atomless while (2.6) (together with Proposition 2.2 (a)) guarantees that all coinfinite supersets of $A_{0}$ are small and hence not $d$-simple.

CoROLlary 2.8. (Lerman, Shore, Soare). The set of degrees containing a coinfinite r.e. set with no d-simple superset is exactly $\boldsymbol{H}_{1}$.

Proof. For any $\boldsymbol{b} \in \boldsymbol{H}_{1}$, there exists $B \subset_{m} A_{0}$ of degree $\boldsymbol{b}$ by Lerman [6]. Now every coinfinite superset $C$ of $B$ is small because $B \subset_{m} A_{0}$ implies $\bar{A}_{0} \nsubseteq^{*} C$, so $C \subset A_{i}$ for some $i$ by (2.7). Thus, by Proposition 2.2 (a) $B$ has no $d$-simple (or even nonsmall) superset.

On the other hand if $\operatorname{deg}(B) \notin \boldsymbol{H}_{1}$ then $B$ has a $d$-simple superset $D$. By $\left[10\right.$, p. 306] there is a recursive array $\left\{W_{f(n)}\right\}_{n \in \omega}$ of disjoint finite sets with union $N$ and such that $\left|W_{f(n)} \cap \bar{B}\right|>n$ for all $n$. We build $D \supseteq B$ to satisfy each positive requirement $P_{n}$ of Theorem 1.7 (and thus be $d$-simple) by allowing any $x \in \bigcup_{m \geqq n} W_{f(n)}$ to serve as the witness for $P_{n}$. Since $\bigcup_{m<n} W_{f(n)}$ is finite, almost every element $x \in \bar{B}$ may serve as a witness for $P_{n}$ so $P_{n}$ is satisfied, yet at most $n$ members of $W_{f(n)}$ are enumerated in $D$ so $W_{f(n)} \cap \bar{D} \neq \phi$ and $\bar{D}$ is infinite.

3. On closure properties of $d$-simple sets. Many classes of simple sets such as simple sets, hypersimple sets, and $h h$-simple sets (although not $r$-maximal sets) are closed under intersection [14, pp. 122, 156, 251] and thus together with the cofinite sets form a filter in $\mathscr{E}$. This is not true for the $d$-simple sets. Indeed we show that there are $d$-simple sets $C$ and $D$ such that $(C \cap D) \subset_{s}(C \cup$ $D) \subset_{\infty} N$, and thus $C \cap D$ is not $d$-simple. We also show that the 
relation " $d$-simple in" is not transitive in contrast to the relation "simple in."

To prepare for these proofs we review Lachlan's strategy [3, p. 134] for ensuring $B \subset_{s} A$. Fix a recursive listing $\left\{\left\langle U_{i}, V_{i}\right\rangle\right\}_{i \in \omega}$ of all pairs of (r.e.) sets, and a simultaneous enumeration of these. We must meet for each $i$ the negative requirement,

$$
N_{i}: V_{i} \supseteqq U_{i} \cap(A-B) \Longrightarrow\left(U_{i}-A\right) \cup V_{i} \text { is r.e. }
$$

To accomplish this we attempt to enumerate a set $T_{i}$ such that if $V_{i} \supseteqq U_{i} \cap(A-B)$ then

$$
T_{i} \subseteq U_{i} \& T_{i} \supseteqq U_{i}-A \&\left(T_{i}-V_{i}\right) \cap B=* \phi,
$$

so that $T_{i} \cup V_{i}={ }^{*}\left(U_{i}-A\right) \cup V_{i}$ and the conclusion of (3.1) is satisfied.

Since $B \subset A$ we may assume that every element $x \in B$ is enumerated in $A$ first. To control the enumeration of $T_{i}$ we have a movable marker $\Gamma_{i}$ whose position at the end of stage $s, \Gamma_{s}^{i}$, is the least $x \in\left(T_{i}^{s}-V_{i}^{s}\right) \cap\left(A^{s}-B^{s}\right)$ if $x$ exists, and $s$ otherwise. At stage $s+1:$ (1) in defining $B^{s+1}$ all $x \in\left(T_{i}^{s}-V_{i}^{s}\right) \cap\left(A^{s+1}-B^{s}\right)$ are restrained with priority $N_{i}$ from entering $B$, and $N_{i}$ is injured if some $x$ which it restrains enters $B$ (say because of a positive requirement of higher priority); (2) after $A^{s+1}$ and $B^{s+1}$ are defined then every $x \in U_{i}^{s}-A_{s+1}$ such that $x \leqq \Gamma_{i}^{s}$ is enumerated $T_{i}$. Clearly $T_{i} \subseteq U_{i}$. Now if $V_{i} \nsupseteq T_{i} \cap(A-B)$ then $V_{i} \nsupseteq U_{i} \cap(A-B)$ so (3.1) is automatically satisfied and furthermore $\lim _{s} \Gamma_{i}^{s}<\infty$, so $T_{i}$ is finite and finitely many $x$ are ever restrained by $N_{i}$. If $V_{i} \supseteqq T_{i} \cap(A-B)$ then $\lim _{s} \Gamma_{i}^{s}=\infty$, so the first two clauses of (3.2) are met and every element $x$ is restrained by $N_{i}$ for at most finitely many stages. Furthermore, if $N_{i}$ is injured at most finitely often then the third clause of (3.2) is also met and thus requirement $N_{i}$ is met.

THEOREM 3.1. The d-simple sets are not closed under intersection.

Proof. We shall construct $d$-simple sets $C$ and $D$ such that $(C \cap D) \subset_{s}(C \cup D) \subset_{\infty} N$, whence $C \cap D$ is small and hence not $d$ simple. Let $\left\{\left\langle X_{e}, Z_{e}\right\rangle\right\}_{e \in \omega}$ and $\left\{\left\langle U_{e}, V_{e}\right\rangle\right\}_{e \in \omega}$ be two listings of all pairs of (r.e.) sets, and fix a simultaneous enumeration of these. To make $C$ and $D d$-simple it suffices to meet the positive requirement $P_{e}$ of (1.3) with $C$ in place of $A$ namely,

$$
P_{e}^{\sigma}:\left(Z_{e}-X_{e}\right) \text { infinite } \Longrightarrow(\exists x)(\exists s)\left[x \in\left(Z_{e}^{s}-X_{e}^{s}\right) \cap\left(C^{s+1}-C^{s}\right)\right],
$$

and similarly $P_{e}^{D}$ with $D$ in place of $C$. Let $A=C \cup D$ and $B=C \cap D$. To insure $B C_{s} A$ we simply meet for each $i$ requirement $N_{i}$ of 
(3.1). The priority ranking of requirements is $\cdots, N_{e}, P_{e}^{C}, P_{e}^{D}, \cdots$. There are no restrictions on an element $x$ first entering $C \cup D$ but once there it may not enter $C \cap D$ for some positive requirement until it is unrestrained by all negative requirements of higher priority.

Stage $s=0 . \quad$ Set $C^{0}=D^{0}=\phi$.

Stage $s+1$. Given $C^{s}$ and $D^{s}$ set $A^{s}=C^{s} \cup D^{s}$ and $B^{s}=C^{s} \cap D^{s}$, and define $T_{i}^{s}$ as above. Choose the positive requirement of highest priority which has never received attention and such that

$$
\begin{aligned}
& (\exists x)\left[x \in\left(Z_{e}^{s}-X_{e}^{s}\right) \& 3 e<x\right. \\
& \quad \& \quad \neg(\exists i \leqq e)\left[x \in\left(T_{i}^{s}-V_{i}^{s}\right) \cap\left(A^{s}-B^{s}\right)\right] .
\end{aligned}
$$

Choose $x$ minimal for $e$. Now $P_{e}$ receives attention and we enumerate $x$ in $C$ if $P_{e}$ is $P_{e}^{C}$ and in $D$ if $P_{e}$ is $P_{e}^{D}$. If $e$ fails to exist do nothing. Let $C=\mathrm{U}_{s} C^{s}$ and $D=\mathrm{U}_{s} D^{s}$.

\section{Lemma 1. $(C \cap D) \subset_{s}(C \cup D) \subset_{\infty} N$.}

Proof. Note that $(C \cup D) \subset_{\infty} N$ by the second clause of (3.3) and the fact that $P_{e}^{C}$ or $P_{e}^{D}$ contributes at most one element to $C \cup D$. Now by the third clause of (3.3) $N_{i}$ is injured by $P_{e}$ only if $e<i$. Thus, for each $i, N_{i}$ is injured only finitely often and $T_{i}$ satisfies the third clause of (3.2). Now if $V_{i} \supseteqq U_{i} \cap(A-B)$ then $T_{i}$ satisfies (3.2) and $N_{i}$ is met.

\section{LemMa 2. $C$ and $D$ are $d$-simple.}

Proof. Fix $e$. Define $I=\left\{i \leqq e: \lim _{s} \Gamma_{i}^{\delta}<\infty\right\}$. Now $T=U$ $\left\{T_{i}: i \in I\right\}$ is finite. If $i \leqq e$ and $i \notin I$ then $V_{i} \supseteqq T_{i} \cap(A-B)$ so no $x$ is restrained by $N_{i}$ at more than finitely many stages. Thus, for any $x \notin T$ there is a stage $s_{x}$ such that for all $s \geqq s_{x}, x$ is not restrained by any $N_{i}, i \leqq e$. Hence, requirements $P_{e}^{C}$ and $P_{e}^{D}$ are met.

Certain notions of simplicity are transitive when considered in relativized form. For example, if $A$ is simple in $B$ (i.e., $A \subseteq B$ and $B-A$ is infinite and immune) and $B$ is simple in $C$ then $A$ is simple in $C$. This is also true when "simple in" is replaced by "hypersimple in" or " $h h$-simple in" but not for " $r$-maximal in" or for " $d$-simple in." For $B C_{\infty} A$ the definition of $B d$-simple in $A$ is obtained from Definition 1.1 by restricting all the quantifiers $X, Y$, $Z$ in Definition 1.1 to be subsets of $A$. 
TheOREM 3.2. There exist sets $B \subset A \subset_{\infty} N$ such that $B$ is $d$ simple in $A$, and $A$ is $d$-simple but $B$ is not $d$-simple (indeed $\left.B \subset_{8} A\right)$.

Proof. Combine the construction of the preceding theorem with the following extra positive requirements $Q_{e}$ which guarantee that $B$ is $d$-simple in $A$,

$$
Q_{e}:\left(Z_{e}-X_{e}\right) \cap A \text { infinite } \Longrightarrow(\exists x)(\exists s)\left[x \in\left(Z_{e}^{s}-X_{e}^{s}\right) \cap\left(B^{s+1}-B^{s}\right)\right] .
$$

Now as in (1.3) we can take $Y_{e}=\left(X_{e} \cap A\right) \backslash B$. The strategy for meeting $Q_{e}$ is to enumerate in $B^{s+1}$ some $x \in\left(Z_{e}^{s} \cap A^{s}\right)-\left(X_{e}^{s} \cup B^{s}\right)$. Since $Q_{e}$ contributes at most one element to $B$ and since the negative requirements $N_{i}$ permanently restrain only finitely many elements of $A-B$ from entering $B$, these requirements $Q_{e}$ can clearly be combined with all the previous requirements in the proof of Theorem 3.1 to obtain the extra conclusion.

4. Degrees of $d$-simple sets. We know by Corollaries 1.6 and 1.8 that all high degrees and some low degrees contain $d$-simple sets. It is natural to conjecture that there is a $d$-simple set in every nonzero degree since this is true for the other known classes of simple sets which intersect the low degrees. To our surprise we discovered that there is a degree $\boldsymbol{d}>\mathbf{0}$ such that every set of lower degree is not $d$-simple (and indeed is small if coinfinite). Thus $\boldsymbol{H}_{1} \subset \boldsymbol{D}$ and $\boldsymbol{D}$ splits $\boldsymbol{L}_{1}$, and likewise for $\boldsymbol{D}$ replaced by $\boldsymbol{N}$, the degrees containing non-small sets. We do not know whether $\boldsymbol{D} \supseteq \grave{\boldsymbol{L}}_{1}$ or whether $N=D$.

THEOREM 4.1. There exists a simple set $S$ such that every coinfinite set $A$ recursive in $S$ is small and hence not $d$-simple.

Proof. We begin with a broad sketch of the proof and then give the detailed construction. We must make $\bar{S}$ infinite and meet for all $j, a$, and $e$ the requirements,

$$
\begin{aligned}
& P_{j}: W_{j} \cap S \neq \phi \text { if } W_{j} \text { is infinite, and } \\
& R_{a, e}: W_{a}=\{e\}^{S} \text { and } \bar{W}_{a} \text { infinite } \Longrightarrow\left(\exists x_{a, e}\right)\left[W_{a} \subset_{s} X_{a, e} \subset_{\infty} N\right] .
\end{aligned}
$$

Given the hypotheses of $R_{a, e}$ we attempt to satisfy its conclusion as in $\S 3$ by meeting for every $i$ the negative requirement $N_{i}$ of (3.1) with $B$ and $A$ replaced by $W_{a}$ and $X_{a, e}$ respectively. Namely, requirement

$$
N_{a, e, i}: V_{i} \supseteqq U_{i} \cap\left(X_{a, e}-W_{a}\right) \Longrightarrow\left(U_{i}-X_{a, e}\right) \cup V_{i} \text { is r.e., }
$$


where $\left\{\left\langle U_{i}, V_{i}\right\rangle\right\}_{i \in \omega}$ is a recursive list of all pairs of (r.e.) sets as in $\S 3$. We accomplish this as in (3.2) by attempting to enumerate $T_{a, e, i}$ so that if the hypothesis of (4.1) is satisfied then

$$
T_{a, e, i} \cong U_{i} \& T_{a, e, i} \supseteq U_{i}-X_{a, e} \&\left(T_{a, e, i}-V_{i}\right) \cap W_{a}={ }^{*} \phi,
$$

so that $T_{a, e, i} \cup V_{i}={ }^{*}\left(U_{i}-X_{a, e}\right) \cup V_{i}$ and the conclusion of (4.1) is satisfied. Define the recursive functions,

$$
\begin{gathered}
u(e, x, s)=\left\{\begin{array}{cc}
\min \left\{z:\{e\}_{s}^{S_{s}[z]}(x)\right. & \text { is defined }\} \text { if } z \text { exists } \\
-1 & \text { otherwise }
\end{array}\right. \\
l(a, e, s)=\max \left\{x:(\forall y \leqq x)\left[W_{a, s}(y)=\{e\}_{s}^{S_{s}}(y)\right]\right\}
\end{gathered}
$$

The main obstacle in achieving $W_{a} \subset_{s} X_{a, e} \subset_{\infty} N$ is that unlike Theorem 3.1 where we controlled $B$, here $W_{a}$ is being enumerated by the "opponent" [4] and so after we enumerate an element $x \in$ $T_{a, e, i}$, the opponent may enumerate $x$ in $W_{a}$ before $x$ appears in $V_{i}$ thereby jeopardizing the final clause of (4.2). To overcome this obstacle and meet requirement $R_{a, e}$ in case $W_{a}=\{e\}^{S}$ and $\bar{W}_{a}$ is infinite we wait for some $x<l(a, e, s)$, such that $x \notin W_{a}^{s} \cup X_{a, e}^{s}$ and we assign a certain marker $\Lambda_{a, e, n}$ to $x$ with the intention that the final positions $\left\{\Lambda_{a, n}^{\omega}\right\}_{n \in \omega}$ of the markers will constitute $\bar{X}_{a, e}$ thereby ensuring $X_{a, e} \subset_{\infty} N$. Now if the opponent enumerates $x$ in $W_{a}$, say at stage $t+1>s$, while $S_{t}[u]=S_{s}[u]$, where $u=u(e, x, s)$ then

$$
W_{a}^{t+1}(x)=1 \text { and }\{e\}_{t}^{S_{t}}(x)=0 .
$$

We then preserve $S_{t}[u]$ with priority $R_{a, e}$ thereby preserving (4.3) and ensuring that $W_{a} \neq\{e\}^{S}$. This negative restraint for $R_{a, e}$ can be injured only by a positive requirement $P_{j}$ such that $j\langle\langle a, e\rangle$. (Let $\Lambda_{a, e, n}^{s}$ denote the position of marker $\Lambda_{a, e, n}$ at the end of stage s.) Hence, for almost every $x=\Lambda_{a, e, n}^{s}$ we can safely assume that $x$ will remain in $\bar{W}_{a}$ until we enumerate in $S$ some $y \leqq u(e, x, s)$.

Corresponding to each negative requirement $N_{a, e, i}$ we have a "gate" $G_{a, e, i}$ as in Lerman's pinball machine model [6]. The gates are arranged in ascending order according to index so $G_{a, e, i}$ lies below $G_{a^{\prime}, e^{\prime}, i^{\prime}}$ just if $\langle a, e, i\rangle\left\langle\left\langle a^{\prime}, e^{\prime}, i^{\prime}\right\rangle\right.$.

Now suppose that requirement $P_{j}$ wishes at stage $s+1$ to enumerate some element $y$ into $S$. We first consider all $x$ such that $y \leqq u(e, x, s)$, since such $x$ may enter $W_{a}$ when we put $y$ into $S$. If some such $x=\Lambda_{a, e, n}^{s}$ for some $\langle a, e, n\rangle \leqq j$, then we do nothing since $P_{j}$ does not have enough priority to move marker $\Lambda_{a, e, n}$. If there is no such $\langle a, e, n\rangle$ (and a few other conditions below are satisfied), then we appoint $y$ as a follower of $P_{j}$ and we attempt to let $y$ pass through all gates of higher priority than $P_{j}$, namely gates $G_{\langle a, e, i\rangle},\langle a, e, i\rangle \leqq j$. 
If the follower $y$ eventually reaches gate $G_{a, e, i}$ at some stage $t+1 \geqq s+1$ we enumerate in $X_{a, e}$ all elements $x \in E(a, e, i, y, j)$, which is a certain set defined at stage $t+1$ and consisting of most elements $x \in T_{a \cdot e i}^{t+1}-W_{a}^{t}$ but excluding $\left\{\Lambda_{a \text { e } e_{i}}^{t}:\langle a, e, n\rangle \leqq j\right\}$. Follower $y$ is later released by $G_{a, e, i}$ at some stage $v+1 \geqq t+1$ if $E(a, e, i$, $y, j) \subseteq V_{i}^{v}$, whereupon $y$ passes to the next lower gate. The point is that no $x \in E(a, e, i, y, j)$ can violate the last clause of (4.2) if $y$ is later enumerated in $S$ because $x \in V_{i}$ already.

Now follower $y$ is eventually either released by all gates and enters $S$ at some stage $w \geqq v+1$, or $y$ is cancelled, or $y$ is a permanent resident of some gate $G_{a, e, i}$. In the latter case $V_{i} \nsupseteq$ $T_{a, e, i} \cap\left(X_{a, e}-W_{a}\right), T_{a, e, i}$ is finite and so there are finitely many permanent residents of $G_{a, e, i}$.

To see that this strategy succeeds in meeting $N_{a, e, i}$ we need to know that no new $x \notin E(a, e, i, y, j)$ is enumerated in $T_{a, e, i}$ between stages $v+1$ and $w$. This requires not just a single set $T_{a, e, i}$ but an infinite list of candidates $\left\{T_{a, e, i, p}\right\}_{p \in \omega}$, such that $T_{a . e . i . p}$ will be the true $T_{a, e, i}$ satisfying (4.2) just if $p$ is the canonical index of the finite set of permanent residents at gates $G_{a^{\prime}, e^{\prime}, i^{\prime}},\left\langle a^{\prime}, e^{\prime}, i^{\prime}\right\rangle \leqq\langle a, e, i\rangle$. (As in Rogers $[13, \mathrm{p} .70]$ let $D_{p}$ denote the finite set whose canonical index is $p$.)

If $y$ and $y^{\prime}$ are followers of $P_{j}$ and $P_{j^{\prime}}$ respectively we say that $y^{\prime}$ has lower order than $y$ if $j<j^{\prime}$ or if $j=j^{\prime}$ and $y^{\prime}$ was appointed after $y$ was appointed. If $y$ and $y^{\prime}$ are followers at stage $s$ we shall arrange that $y<y^{\prime}$ iff $y^{\prime}$ has lower order than $y$. A follower $y$ of $P_{j}$ once cancelled can later be appointed to follow $P_{j^{\prime}}$ only if $j^{\prime}<j$.

\section{CONSTRUCTION.}

Stage $s=0$. Do nothing. Set $Z^{0}=\phi$ for all sets $Z, M^{0}=-1$ for all movable markers $M$, and $r(a, e, 0)=-1$ for all $a$ and $e$.

Stage $s+1$. Perform in order the following steps.

Step 1. For all $\langle a, e\rangle \leqq s$, if either $r(a, e, s)=-1$ or there is a $y \in S^{s}-S^{s-1}$ such that $y<r(a, e, s)$, set $r^{\prime}(a, e, s)=-1$. Otherwise, set $r^{\prime}(a, e, s)=r(a, e, s)$ (whether or not $\left.\langle a, e\rangle \leqq s\right)$. If $r^{\prime}(a, e$, $s)=-1$, and there exists $x \in W_{a}^{s}$ such that $\{e\}_{s}^{S_{s}}(x)=0$, choose $x$ minimal for $a$ and $e$ and set $r(a, e, s+1)=u(e, x, s)$. Otherwise, set $r(a, e, s+1)=r^{\prime}(a, e, s)$. If $r(a, e, s+1) \neq r^{\prime}(a, e, s+1)=-1$, then cancel every follower of every requirement $P_{j}, j \geqq\langle a, e\rangle$.

Step 2. If $r(a, e, s+1)\rangle-1$ or $\langle a, e\rangle\rangle s$, let $L_{a, e}^{s+1}=\phi$ and 
$\Lambda_{a, e, n}^{s}=-1$ for all $n$. If $r(a, e, s+1)=-1$ let $\Lambda_{a, e, n}^{s+1}$ be the $n$th element of the set

$$
L_{a, e}^{s+1}=\left\{x: x \in W_{a}^{s} \cup X_{a, e}^{s} \& x<l(a, e, s)\right\}
$$

if such element exists and $\Lambda_{a \in n}^{s+1}=-1$ otherwise. Define

$$
F(j, s+1)=\left\{x: x=\Lambda_{a, e n}^{s+1} \&\langle a, e, n\rangle \leqq j\right\} .
$$

If either $F(j, s+1) \neq F(j, s)$ or $u(e, x, s) \neq u(e, x, s-1)$ for some $x \in \bigcup_{t \leqq s+1} F(j, t)$ then cancel every follower of $P_{j}$, for all $j^{\prime} \geqq j$.

Step 3. If follower $y$ of requirement $P_{j}$ is at gate $G_{a, e, i}$ and $u(e, x, s) \neq u(e, x, s-1)$ for some $x \in E(a, e, i, y, j)$ then cancel all followers $z \geqq y$.

Step 4. Define

$$
\Gamma_{a, i}^{s+1}= \begin{cases}(\mu x)\left[x \in\left(X_{a, e}^{s}-W_{a}^{s}\right) \cap\left(T_{a, e i}^{s}-V_{i}^{s}\right)\right] & \text { if } x \text { exists, } \\ s & \text { otherwise. }\end{cases}
$$

Define

$H(a, e, i, s+1)=\left\{y: y\right.$ is a follower now at a gate $G_{a^{\prime}, e^{\prime}, i^{\prime}}$ for some $\left.\left\langle a^{\prime}, e^{\prime}, i^{\prime}\right\rangle \leqq\langle a, e, i\rangle\right\}$.

Choose $p$ such that $D_{p}=H(a, e, i, s+1)$. Let

$$
x=(\mu z)\left[z \leqq \Gamma_{a, e, i}^{s+1} \& z \in\left(U_{i}^{s} \cap L_{a e}^{s+1}\right)-T_{a, e, i, p}^{s}\right] .
$$

Enumerate $x$ in $T_{a, e, i, p}$. If $x$ is not defined do nothing. Let $T_{a, e, i}^{s+1}=$ $\bigcup_{p} T_{a, e, i, p}^{s+1}$.

Step 5. Requirement $P_{j}$ requires attention if $W_{j, s} \cap S_{s}=\phi$, and one of the following two conditions holds.

Condition 1. A follower $y$ of $P_{j}$ now at some gate $G_{a, e, i}$ is released by $G_{a, e, i}$ namely

$$
E(a, e, i, y, j) \subseteq V_{i}^{s} \cup W_{a}^{s} .
$$

Condition 2. All existing followers of $P_{j}$ currently reside at gates, and there exists $y \in W_{j}^{s}$ such that $y>2 j, y>$ all previously appointed followers of requirements $P_{j^{\prime}}, j^{\prime} \leqq j$, and

$$
(\forall x)\left[x \in \bigcup_{t \leq s+1} F(j, t) \Longrightarrow u(e, x, s)<y\right],
$$




$$
(\forall a)(\forall e)[\langle a, e\rangle \leqq j \Longrightarrow r(a, e, s+1)<y] .
$$

If no $P_{j}$ requires attention go to step 6 . Otherwise, choose the least $j$ such that $P_{j}$ requires attention and the least $y$ corresponding to $P_{j}$. Cancel all followers $z$ of lower order than $y$, and adopt the first case below which holds.

Case 1. Condition 1 holds. If $\langle a, e, i\rangle=0$ enumerate $y$ in $S$. Otherwise move $y$ to gate $G_{a^{\prime}, e^{\prime}, i^{\prime}}$, the next gate below $G_{a, e, i}$. Let $C$ be the set of all $x$ such that $x \in E\left(a^{\prime}, e^{\prime}, i^{\prime}, y^{\prime}, j^{\prime}\right)$ for some follower $y^{\prime}<y$ now residing at gate $G_{a^{\prime}, e^{\prime}, i^{\prime}}$ and following some $P_{j^{\prime}}$. Define

$$
\begin{aligned}
E\left(a^{\prime}, e^{\prime}, i^{\prime}, y, j\right)= & \left\{x: x \in T_{a^{\prime}, e^{\prime}, i^{\prime}}^{s+1}-\bigcup_{t \leq s+1} F(j, t)\right\} \\
& -\left\{x: x \in C \& u\left(e^{\prime}, x, s\right)<y\right\} .
\end{aligned}
$$

Enumerate in $X_{a^{\prime}, e^{\prime}}$ all $x \in E\left(a^{\prime}, e^{\prime}, i^{\prime}, y, j\right)$.

Case 2. Condition 2 holds. Appoint $y$ to follow $P_{j}$. Place $y$ at gate $G_{a^{\prime}, e^{\prime}, i^{\prime}}$, where $j=\left\langle a^{\prime}, e^{\prime}, i^{\prime}\right\rangle$, and proceed as in Case 1 .

Step 6. If $x \in W_{a}^{s}-W_{a}^{s-1}$ enumerate $x$ in $X_{a, e}$.

This completes the construction at stage $s+1$. Define $Z=\mathrm{U}_{s} Z^{s}$ for each set $Z$ mentioned above.

LEMMA 1. For all $e$ and $x, u(e, x)=\lim _{s} u(e, x, s)$ exists, and $S$ is low (i.e., $S^{\prime} \equiv_{T} \phi^{\prime}$ ).

Proof. This follows automatically from the restraint function $r$ as in [18, Theorem 4.1 and Remark 4.5]. Fix $e$ and $x$. Uniformly effectively in $e$ and $x$ we can choose a such that $W_{a}=\{0\}$ and $b$ such that for every $\sigma \in \omega^{<\omega},\{b\}_{s}^{\sigma}(0)=0$ iff $\{e\}_{s}^{\sigma}(x)$ is defined. Some $y<r(a, b, s)$ can be put into $S$ at stage $s+1$ by $P_{j}$ only if $j\langle\langle a, b\rangle$, but each $P_{j}$ contributes at most one such element to $S$, so $\lim _{s} r(a$, $b, s)$ exists. Now if $\{e\}_{s}^{S_{s}}(x)$ is defined for infinitely many $s$, then $\{b\}_{s}^{S_{s}}(0)=0$ for infinitely many $s$ and hence for cofinitely many $s$ by step 1 and the definition of $r$. Thus, $\lim _{s} r(a, b, s)>-1$ and $\{e\}^{S}(x)$ is defined. Hence, $u(e, x)=\lim _{s} u(e, x, s)$ exists and is recursive in $\phi^{\prime}$ by the Limit Lemma [15, p. 29]. Finally, $S$ is low since $e \in S^{\prime}$ iff $u(e, e)>-1$.

LEMMA 2. For each gate $G_{a, e, i}$ there are at most finitely many followers $y$ which reside permanently at $G_{a, e, i}$.

Proof. Suppose follower $y$ of $P_{j}$ resides at $G_{a, e, i}$ at all stages 
$\geqq s_{0}$. Then $E(a, e, i, y, j) \nsubseteq V_{i} \cup W_{a}$ so $\left(X_{a, e}-W_{a}\right) \cap T_{a, e, i} \nsubseteq V_{i}$, and hence $T_{a, e, i}$ is finite because of step 4 . Choose $s_{1} \geqq s_{0}$ such that $T_{a, e i}^{s}=T_{a, e, i}$ and $u(e, x, s)=u(e, x)$ for all $x \in T_{a, e, i}$ and $s \geqq s_{1}$. Let $z_{1}=\max \left\{u(e, x): x \in T_{a, e, i}\right\}$.

Now suppose for a contradiction that $G_{a, e, i}$ has infinitely many permanent residents. For each $m>1$ let $y_{m}>z_{1}$ be a permanent resident of $G_{a, e, i}$ which follows some $P_{j_{m}}$ and arrives at $G_{a, e, i}$ at some stage $s_{m}+1>s_{1}+1$. Let $C_{m}$ be defined for $y_{m}$ as in Case 1 of step 5 (with $a, e, i$ in place of $a^{\prime}, e^{\prime}, i^{\prime}$ ). Now since $y_{m}$ is a permanent resident of $G_{a, e, i}, E\left(a, e, i, y_{m}, j_{m}\right) \neq \phi$, and so must contain an element $v_{m} \in T_{a, e, i}-C_{m}$. But the definition of $C_{m}$ implies that $v_{m_{1}} \neq v_{m_{2}}$ for $m_{1} \neq m_{2}$, so there can be finitely many such elements $y_{m}$ because $T_{a, e, i}$ is finite.

\section{LEMMA 3. $S$ is simple.}

Proof. First $\bar{S}$ is infinite since by step 5 Case 2 if $y$ is appointed to follow $P_{j}$ then $y>2 j$. It remains to show that for all $j$ requirement $P_{j}$ is met. Fix $j$ and assume that for all $j^{\prime}<j, P_{j^{\prime}}$ is met and receives attention at most finitely often. Choose $s_{0}$ such that no $P_{j^{\prime}}, j^{\prime}<j$, requires attention after stage $s_{0}$. Now we can choose $s_{1}>s_{0}$ such that $r(a, e, s)=r\left(a, e, s_{1}\right)$ for all $s \geqq s_{1}$ and all $\langle a, e\rangle \leqq j$, because $P_{j^{\prime}}$ can contribute to $S$ an element $y \leqq r(a, e, s)$ only if $j^{\prime}<\langle a, e\rangle$.

Next we choose $s_{2}>s_{1}$ such that $F\left(j, s_{2}\right)=F(j, s)$ for all $s \geqq s_{2}$. To see that this is possible fix $\langle a, e, n\rangle \leqq j$ and assume that for all $n^{\prime}<n$, marker $\Lambda_{a, e, n}$, does not move after stage $v>s_{1}$. Suppose $\Lambda_{a, e, n}^{s}=x>-1$ for some $s>v$, where $x$ is minimal for all $s>v$. Then $r(a, e, t)=-1$ for all $t \geqq s_{1}$. Hence, by the choice of $s_{0}$ and cancellation of step 2 and (4.4), $u(e, x, t)=u(e, x, s)$ for all $t \geqq s$, and $\{e\}_{t}^{S_{t}}(x)=0$ for all $t \geqq s$. Now $x$ cannot be enumerated in $W_{a}$ after stage $s$ else step 1 later applies to $\langle a, e\rangle$ contrary to the choice of $s_{1}$. But $x$ cannot be enumerated in $X_{a, e}$ at a stage $t+1>s$ else step 5 applies to some $P_{j^{\prime}}, j^{\prime}\langle\langle a, e, n\rangle$, contrary to choice of $s_{0}$. Thus, $\Lambda_{a, e, n}^{t}=x$ for all $t \geqq s$, and $s_{2}$ exists. Set $F(j)=\bigcup_{s} F(j, s)=$ $\bigcup_{s \geqq s_{2}} F(j, s)$.

Choose $s_{3} \geqq s_{2}$ such that $u(e, x)=u(e, x, s)$ for all $s \geqq s_{3}, e \leqq j$, and $x \in F(j)$. Now after stage $s_{3}$ the cancellation of steps 1 and 2 cannot apply to $P_{j}$. By Lemma 2 there can be at most finitely many permanent residents $y_{1}, \cdots, y_{m}$ at gates $G_{a, e, i},\langle a, e, i\rangle \leqq j$. Choose $z_{1}>u(e, x)$ for every $x \in \cup\left\{E\left(a, e, i, y_{k}, j_{k}: 1 \leqq k \leqq m\right)\right\}$, where $y_{k}$ permanently follows $P_{j_{k}}$. Choose $s_{4} \geqq s_{3}$ such that $u(e, x, s)=u(e, x)$ for all $s \geqq s_{4}$ and $x \leqq z_{1}$, and such that each $y_{k}, k \leqq m$, has reached its final gate position by stage $s_{4}$. 
Now suppose that $W_{j}$ is infinite and $W_{j} \cap S=\phi$. Choose a stage $s_{5}+1>s_{4}+1$ at which some follower $y$ of $P_{j}$ is appointed and such that no follower $y^{\prime}<y$ ever receives attention after stage $s_{5}+1$. Now $y$ cannot be cancelled at step 3 or step 5 by choice of $s_{4}$ and $s_{5}$ respectively. Note that when $y$ reaches gate $G_{a, e, i}$, all residents of $G_{a, e, i}$ must be permanent. Hence, $y$ can never be cancelled. But $y$ is not a permanent resident of any gate $G_{a, e, i},\langle a, e, i\rangle \leqq$ $j$ so $y$ eventually enters $S$. Hence, requirement $P_{j}$ is met and receives attention at most finitely often.

LEMMA 4. For all $a$ and $e$ requirement $R_{a, e}$ is met.

Proof. Assume $W_{a}=\{e\}^{S}$ and $\bar{W}_{a}$ is infinite. Hence, by the proof of Lemma 3, for all $a, e$, and $n, \Lambda_{a, e, n}^{\omega}=\lim _{s} \Lambda_{a, e, n}^{s}$ exists and $\Lambda_{a, e, n}^{\omega}>-1$. Hence, $X_{a, e} \subset_{\infty} N$, and $W_{a} \subset X_{a, e}$ by step 6 . To prove $W_{a} \subset_{s} X_{a, e}$ we must verify that requirement $N_{a, e, i}$ is met for all $i$.

Fix $\langle a, e, i\rangle$. Now assume the hypothesis of (4.1), namely $V_{i} \supset$ $U_{i} \cap\left(X_{a, e}-W_{a}\right)$. Then $V_{i} \supset T_{a, e, i} \cap\left(X_{a, e}-W_{a}\right)$, and hence $\lim _{s} \Gamma_{a, e, i}^{s}=$ $\infty$. By Lemma 2 choose $p$ such that

$$
\begin{gathered}
D_{p}=\{y: y \text { is a permanent resident of some gate } \\
\left.G_{a^{\prime}, e^{\prime}, i^{\prime}},\left\langle a^{\prime}, e^{\prime}, i^{\prime}\right\rangle<\langle a, e, i\rangle\right\} .
\end{gathered}
$$

We shall show that $T_{a, e, i, p}$ satisfies (4.2).

We call stage $s+1$ a nondeficiency stage of the construction if some requirement $P_{j}$ receives attention or is cancelled at stage $s+1$ and for all $j^{\prime} \leqq j$ no follower $y$ of $P_{j^{\prime}}$ receives attention or is cancelled at any stage $t>s+1$. Note that there are infinitely many nondeficiency stages, and at all sufficiently large nondeficiency stages $s, H(a, e, i, s)=D_{p}$ because whenever a follower is cancelled, all followers of lower order are cancelled, so any follower existing at stage $s$ remains in existence and in its current position at all later stages. Therefore, each $x \in U_{i}-X_{a, e}$ will be eventually enumerated in $T_{a, e, i, p}$ at step 4 of some nondeficiency stage.

Thus, $T_{a, e, i, p}$ satisfies the first two clauses of (4.2). To see that it satisfies the final clause also, choose $s_{0}$ such that all followers $y \in D_{p}$ are in their final gate positions by stage $s_{0}$, no requirement $P_{j}, j<\langle a, e, i\rangle$, receives attention at any stage $s \geqq s_{0}$, and $r(a, e, s)=$ -1 for all $s \geqq s_{0}$.

Now suppose $x \in T_{a, e, i, p}^{s+1}-T_{a, e, i, p}^{s}$ for some $s>s_{0}$. Then $x \in L_{a, e}^{s+1}$ and $\{e\}_{s}^{S_{s}}(x)=0$. Now if $x \in W_{a}^{t+1}-W_{a}^{t}$ for some $t+1>s+1$ then $y \in S^{v+1}-S^{v}$ for some $y \leqq u=u(e, x, v)$, and $v$ such that $s \leqq v \leqq t$ else $S_{t}[u]=S_{s}[u],\{e\}_{t}^{S_{t}}(x)=0$, and step 1 applies to $\langle a, e\rangle$ after stage $t$ contrary to the choice of $s_{0}$. Let $y$ be the first such follower so 
that $v$ is minimal. Then $u(e, x, s)=u(e, x, v)$. Suppose $y$ follows $P_{j}$. Then $\left.j\right\rangle\langle a, e, i\rangle$ by choice of $s_{0}$. Now $y$ cannot have been at a gate $G_{a^{\prime}, e^{\prime}, i^{\prime}}$ for $\left\langle a^{\prime}, e^{\prime}, i^{\prime}\right\rangle \leqq\langle a, e, i\rangle$ at stage $s+1$ because $y \notin D_{p}$. Therefore, $y$ enters $G_{a, e, i}$ at some stage $s_{1}+1$, such that $s \leqq s_{1} \leqq v$.

By step 5 Case $1 x$ is put into $E(a, e, i, y, j)$ at stage $s_{1}+1$ unless either: (1) $x \in \cup\left\{F(j, k): k \leqq s_{1}+1\right\}$; or (2) $u\left(e, x, s_{1}\right)<y$ and $x \in$ $E\left(a, e, i, y^{\prime}, j^{\prime}\right)$ for some follower $y^{\prime}<y$ which rests at $G_{a, e, i}$ at stage $s_{1}+1$ and follows some $P_{j^{\prime}}$. But (1) cannot hold else $u\left(e, x, s_{1}\right)<y$ and hence $u(e, x, v)<y$ by (4.4) and because otherwise $y$ is cancelled before stage $v+1$ according to step 2. (Notice that (4.4) also rules out $y$ being appointed too late.) Likewise, (2) cannot hold else $u\left(e, x, s_{1}\right)<y$ and hence $u(e, x, v)<y$ since otherwise $y$ is cancelled before stage $v+1$ according to step 3 .

Therefore $x \in E(a, e, i, y, j)$ and $x$ must have been released by $G_{a, e, i}$ at some stage $s_{2}+1$ such that $s_{1} \leqq s_{2} \leqq v$ at which time $x \in$ $V_{i}^{s_{a}}$. Therefore $\left(T_{a, e, i, p}-V_{i}\right) \cap A={ }^{*} \phi$ and $T_{a, e, i, p}$ satisfies (4.2) so requirement $N_{a, e, i}$ is met.

5. Final remarks and open questions. In view of the close resemblance between non- $d$-simple sets and small sets we would like to know whether every non- $d$-simple set is small. The obvious attack that $A$ non- $d$-simple via $X$ implies $A \subset_{s} X$ fails. If these classes fail to coincide (as seems likely) is it at least true that $D=$ $N$, the degrees containing non-small sets? The construction and proof in Theorem 4.1 strongly used the fact that $S$ is low. Can this be extended to non-low degrees? In particular, is it true that $\boldsymbol{D} \supset \overline{\boldsymbol{L}}_{1}$ ? Is there any elegant description of $\boldsymbol{D}$ analogous to the definitions of $\boldsymbol{L}_{n}$ and $\boldsymbol{H}_{n}$ ?

A major open question is to find all the invariant classes of degrees and in particular to determine whether $\boldsymbol{H}_{n}$ and $\overline{\boldsymbol{L}}_{n}$ are invariant for every $n$. In particular, is $\bar{L}_{1}$ invariant? If so one should be able to find a condition analogous to "atomless" and carry out the procedure of Lachlan [4] and Shoenfield [16]. After repeated attempts no such condition has emerged. If $\overline{\boldsymbol{L}}_{1}$ is not invariant then one ought to be able to prove using automorphisms that for any invariant class $\boldsymbol{C}$ if $\overline{\boldsymbol{L}}_{2} \varsubsetneqq \boldsymbol{C}$ then $\boldsymbol{C} \cap \boldsymbol{L}_{1} \neq \phi$. To do this one would hope to show that for any coinfinite set $A$ such that $\operatorname{deg}(A) \in$ $\boldsymbol{L}_{2}$ there exists $\Phi \in$ Aut $\mathscr{E}$ such that $\operatorname{deg}(\Phi(A)) \in \boldsymbol{L}_{1}$. However, by [20] this would imply that $\mathscr{L}(A) \cong \mathscr{E}$ for every such $A$ and we have been unable to push Lachlan's construction [2, Theorem 4] to verify this. We do not even know whether for such an $A$ and for any $\exists \forall \exists-B o o l e a n$ algebra $\mathscr{B}[2$, p. 21] there exists $B \supseteqq A$ such that $\mathscr{L}^{*}(B) \cong \mathscr{B}$. This has been verified however [1, Theorem 4.8] for 
coinfinite $A$ satisfying a lowness property similar to $\operatorname{deg}(A) \in \boldsymbol{L}_{2}$.

Finally, we are interested in the role of $d$-simplicity and its stronger versions in classifying the automorphism types of members of $\mathscr{E}$. If $A$ and $B$ are $d$-simple and low is $A$ automorphic to $B$ ? What are sufficient conditions on $A$ and $B$ for (1.5) to hold? Which other definable classes $\mathscr{C} \subseteq \mathscr{E}$ besides maximal sets and infinite, coinfinite sets constitute orbits under Aut $\mathscr{E}$ ?

\section{REFERENCES}

1. V. L. Bennison and R. I. Soare, Some lowness properties and computational complexity sequences, Theoretical Computer Science, 6 (1978), 233-254.

2. A. H. Lachlan, On the lattice of recursively enumerable sets, Trans. Amer. Math. Soc., 130 (1968), 1-37.

3. - The elementary theory of recursively enumerable sets, Duke Math. J., 35 (1968), 123-146.

4. - Degrees of recursively enumerable sets which have no maximal superset, J. Symbolic Logic, 33 (1968), 431-443.

5. - On some games which are relevant to the theory of recursively enumerable sets, Ann. of Math., (2) 91 (1970), 291-310.

6. M. Lerman, Some theorems on r-maximal sets and major subsets of recursively enumerable sets, J. Symbolic Logic, 36 (1971), 193-215.

7. - Admissible ordinals and priority arguments, Proceedings of the Cambridge Summer School in Logic 1971, Lecture Notes in Mathematics No. 337, SpringerVerlag, 1973.

8. M. Lerman and R. I. Soare, A decidable fragment of the elementary theory of the lattice of recursively enumerable sets, Trans. A.M.S., 257 (1980), 1-37.

9. M. Lerman, R. A. Shore, and R. I. Soare, r-Maximal major subsets, Israel J. Math., 31 (1978), 1-18.

10. D. A. Martin, Classes of recursively enumerable sets and degrees of unsolvability, Z. Math. Logik Grundlagen Math., 12 (1966), 295-310.

11. E. L. Post, Recursively enumerable sets of positive integers and their decision problems, Bull. Amer. Math. Soc., 50 (1944), 284-316.

12. R. W. Robinson, Simplicity of recursively enumerable sets, J. Symbolic Logic, 32 (1967), 162-172.

13. — A dichotomy of the recursively enumerable sets, Zeitschr. f. Math. Logik und Grundlagen Math., 14 (1968), 339-356.

14. H. Rogers, Jr., Theory of Recursive Functions and Effective Computability, McGraw-Hill, New York, 1967.

15. J. R. Shoenfield, Degrees of Unsolvability, North-Holland, Amsterdam, 1971.

16. - Degrees of classes of r.e. sets, J. Symbolic Logic, 41 (1976), 695-696.

17. R. I. Soare, Automorphisms of the lattice of recursively enumerable sets. Part

I: Maximal sets, Annals of Mathematics, 100 (1974), 80-120.

18. - The infinite injury priority method, J. Symbolic Logic, 41 (1976), 513-530.

19. Computational complexity, speedable and levelable sets, J. Symbolic Logic., 42 (1977), 545-563.

20. - Automorphisms of the lattice of recursively enumerable sets. Part II, Low sets, to appear.

21. M. Stob, The structure and elementary theory of the recursively enumerable sets, Ph. D. Dissertation, University of Chicago, 1979.

22. C. E. M. Yates, Three theorems on the degree of recursively enumerable sets, Duke Math. J., 32 (1965), 461-468. 
Received January 15, 1978. These results were presented to the American Mathematical Society at a Special Session for Recursion Theory during the Toronto meeting in August, 1976. This research was supported by NSF Grants MCS 76-07258 and MCS 76-07033 A01 respectively. The authors are grateful to Carl G. Jockusch and Richard Shore for helpful discussions and suggestions.

The University of Connecticut STORRS, CT 06268

AND

The University of Chicago

Chicago, IL 60637 



\section{PACIFIC JOURNAL OF MATHEMATICS}

\section{EDITORS}

DONALD BABBITT (Managing Editor)

University of Galifornia

Los Angeles, California 90024

HUGo RossI

University of Utah

Salt Lake City, UT 84112

C. C. MOORE AND ANDREW OGG

University of California

Berkeley, CA 94720

\section{J. DUGUNDJI}

Department of Mathematics University of Southern California Los Angeles, California 90007

R. FINN AND J. MILGRAM Stanford University Stanford, California 94305

ASSOCIATE EDITORS
E. F. BECKENBACH
B. H. NeumanN
F. WoLF
K. YosHIDA

\section{SUPPORTING INSTITUTIONS}

UNIVERSITY OF BRITISH COLUMBIA UNIVERSITY OF SOUTHERN CALIFONIA CALIFORNIA INSTITUTE OF TECHNOLOGY UNIVERSITY OF CALIFORNIA MONTANA STATE UNIVERSITY STANFORD UNIVERSITY UNIVERSITY OF HAWAII UNIVERSITY OF TOKYO UNIVERSITY OF NEVADA, RENO UNIVERSITY OF UTAH NEW MEXICO STATE UNIVERSITY WASHINGTON STATE UNIVERSITY OREGON STATE UNIVERSITY UNIVERSITY OF OREGON UNIVERSITY OF WASHINGTON 


\section{Pacific Journal of Mathematics \\ Vol. 87, No. $1 \quad$ January, 1980}

Spiros Argyros, A decomposition of complete Boolean algebras ..........

Gerald A. Beer, The approximation of upper semicontinuous multifunctions

by step multifunctions . . . . ....................

Ehrhard Behrends and Richard Evans, Multiplicity theory for Boolean

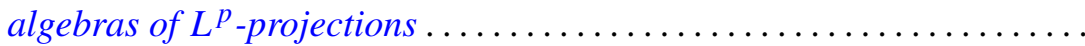

Man-Duen Choi, The full $C^{*}$-algebra of the free group on two

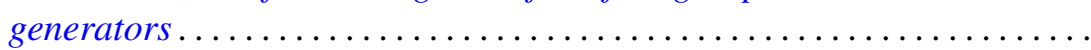

Jen-Chung Chuan, Axioms for closed left ideals in a $C^{*}$-algebra . . . . . . . .

Jo-Ann Deborah Cohen, The strong approximation theorem and locally

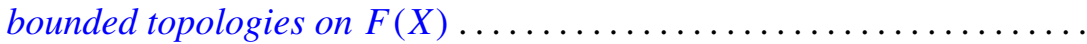

Eugene Harrison Gover and Mark Bernard Ramras, Increasing sequences of Betti numbers............................

Morton Edward Harris, Finite groups having an involution centralizer with

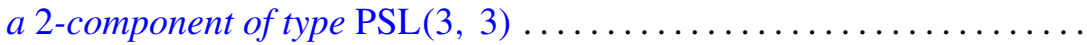

Valéria Botelho de Magalhães Iório, Hopf $C^{*}$-algebras and locally compact

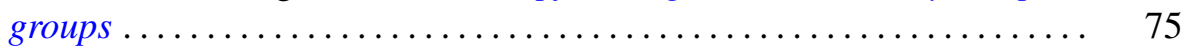

Roy Andrew Johnson, Nearly Borel sets and product measures . . . . . . . . . .

Lowell Edwin Jones, Construction of $Z_{p}$-actions on manifolds . . . . . . . . .

Manuel Lerman and Robert Irving Soare, $d$-simple sets, small sets, and

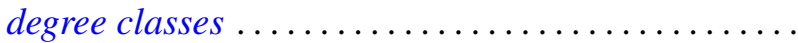

Philip W. McCartney, Neighborly bushes and the Radon-Nikodým property

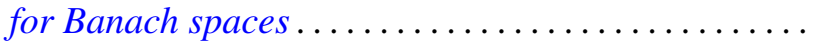

Robert Colman McOwen, Fredholm theory of partial differential equations on complete Riemannian manifolds.

Ernest A. Michael and Carl Preston Pixley, A unified theorem on continuous selections.

Ernest A. Michael, Continuous selections and finite-dimensional sets .

Vassili Nestoridis, Inner functions: noninvariant connected components...

Bun Wong, A maximum principle on Clifford torus and nonexistence of proper holomorphic map from the ball to polydisc.

Steve Wright, Similarity orbits of approximately finite $C^{*}$-algebras . . .

Kenjiro Yanagi, On some fixed point theorems for multivalued

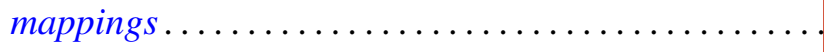

Wieslaw Zelazko, A characterization of LC-nonremovable ideals in commutative Banach algebras 